\title{
Uso de meios digitais, capacidades de inovação e resultados do sojicultor no PR e MATOPIBA
}

Use of digital media, innovation capabilities and results of soybean farmers in PR and MATOPIBA

\author{
Anderson Nora Ribeiro ${ }^{1 *}$; Caetano Haberli Jr. ${ }^{2}$ (1)
}

Received: jun. 7, 2021

Accepted: nov. 23, 2021

${ }^{1 *}$ Microquimica Tradecorp. Gerente de Marketing. Rua Professor Antonio Nogueira Braga, 110, casa 37 - Pq. Rural Faz. Santa Cândida; 13087-601, Campinas, SP, Brasil. <anderphoenix1@ hotmail.com>.

${ }^{2}$ Doutor em Sistemas de Informação. Rua Guarujá, 240 - 13287154, Vinhedo, SP, Brasil.

\author{
(c) (1) \\ Este é um artigo publicado em acesso aberto (Open Access) \\ sob a licença Creative Commons Attribution, que permite uso, \\ distribuição e reprodução em qualquer meio, sem restrições desde \\ que 0 trabalho original seja corretamente citado.
}

\begin{abstract}
Resumo: O Brasil é dos mais relevantes produtores agrícolas do mundo, sendo um fundamental fornecedor de alimentos. O cultivo agrícola de maior destaque no país é a soja, cultura na qual o país é o primeiro produtor e exportador, com altos níveis de produtividade e um sistema de produção baseado em conhecimento científico desenvolvido localmente. O cultivo faz uso intensivo de tecnologias e inovação, e diante da transformação digital pela qual tem passado, o objetivo desta pesquisa foi entender a realidade e perspectivas futuras do produtor rural em termos de busca e utilização de inovações, hábito de uso de ferramentas digitais e sua utilidade na gestão e tomada de decisão na atividade agrícola. Foram entrevistados, por formulário eletrônico e ligações telefônicas, 55 produtores de duas importantes regiões sojícolas, o Centro e Norte do Paraná e a região do MATOPIBA. Os resultados foram analisados por meio de Análises Fatoriais e de Equações Estruturais, a fim de se confirmar as hipóteses ou constructos. O uso de meios digitais nas propriedades teve influência positiva muito significativa na capacidade de inovação, assim como na absorção de inovação. Por sua vez, a capacidade de inovação teve efeito também positivo na geração de vantagens competitivas das propriedades. No entanto, não foi possível comprovar influência da absorção de inovação nas vantagens competitivas. $\mathrm{O}$ aprimoramento e estímulo ao uso dos meios digitais voltados para o agronegócio é importante, e estudos semelhantes serão necessários para gerar informação e aceleração da transformação digital no campo.
\end{abstract}

Palavras-chave: capacidades; meios digitais; inovação; soja.

\begin{abstract}
Brazil is one of the most relevant agriculture producers in the world, been a fundamental food supplier. The crop that stands out is soybean, in which the country is the first producer and exporter, with a higher level of yield and a production system based on locally developed science. Soybean cultivation uses intensive technology and innovation and under the light of digital transformation that has been happening, the objective of this research was to understand the reality and future perspectives of the rural producer in terms of the search and use of innovations, habit of using digital tools and its usefulness in management and decision-making in agricultural activity. Fifty-five growers were interviewed using electronic and telephonic questionnaire, of two important soybean regions, the Center and North of Parana state and MATOPIBA region. The results were analysed using Factorial Analysis and Structural Equations, in order to confirm the hypotheses or constructs. The use of digital media in properties had a significative and positive influence on the capacity for innovation, as well as, on the absorption of innovation. Additionally, the capacity for innovation had also a positive effect on the generation of competitive advantages, but the influence of the absorption of innovation on competitive advantages has not been proven. It is important to improve and push the use of digital media for the agricultural sector and other studies will be necessary, to generate new information and accelerate the digital transformation of the field.
\end{abstract}

Keywords: capacities; digital media; innovation; soybean. 


\section{Introdução}

O agronegócio é um dos setores mais importantes e bem-sucedidos da economia brasileira, representando 21,4\% (RS 1,55 trilhão) do PIB brasileiro, com um Valor Bruto da Produção (VBP) agropecuária de $R \$ 651,5$ bilhões, em 2019. O Brasil é um dos mais importantes produtores globais de diversos produtos agrícolas, entre eles açúcar, café, suco de laranja, milho e, em especial, soja (CNA, 2020).

Segundo dados do Levantamento Sistemático da Produção Agropecuária (LSPA) do Instituto Brasileiro de Geografia e Estatística (IBGE, 2020a), o país cultivou na safra 2019/20, 37.066 .990 ha de soja e colheu 121.386 .542 t do grão. O relatório da Companhia Nacional de Abastecimento (CONAB, 2020) traz previsão ainda mais elevada, atingindo 124.844 .500 t de grãos no referido ano agrícola.

As fontes mostram que o Brasil é o maior produtor mundial da oleaginosa, pois conforme relatório do United States Department of Agriculture (USDA, 2020b), a estimativa para a safra 2020/21 dos Estados Unidos é de 116,15 milhões de toneladas, ante uma produção de 96,67 milhões de toneladas na safra 2019/20. O Brasil também é o maior exportador do grão, o que consolida seu papel de destaque com relação ao cultivo (USDA, 2020a).

A soja é também uma cultura versátil em termos de distribuição geográfica e em perfil de produtores no Brasil. Conforme o Censo Agropecuário de 2017, do IBGE, o país possui 236.245 estabelecimentos produtores de soja (IBGE, 2017). A cultura é produzida em dezenove unidades da Federação e no Distrito Federal. A área média plantada por produtor vai de pequenas áreas de 40 ha e 61 ha, em Santa Catarina e Rio Grande do Sul, até áreas de 2.177ha e 2.806ha por propriedade, no Maranhão e Piauí, respectivamente.

Apesar do cultivo sustentar milhares de pequenos agricultores e agricultores familiares, há que se destacar que grande parte dos resultados do cultivo vem de regiões, onde o mesmo é realizado com alto conhecimento técnico, tecnologia de ponta e, consequentemente, alto investimento, resultando em índices de produtividade equivalentes aos obtidos por sojicultores americanos, conforme dados compilados pela Empresa Brasileira de Pesquisa Agropecuária (EMBRAPA), pela Unidade Descentralizada, Embrapa Soja, em outubro de 2020 (EMBRAPA, 2020), que mostraram produtividade média de 56,32 sc/ $\mathrm{ha}^{-1}\left(3.379 \mathrm{~kg} / \mathrm{ha}^{-1}\right)$ no Brasil, quando nos EUA a média na safra 2020 foi de $53,12 \mathrm{sc} / \mathrm{ha}^{-1}\left(3.187 \mathrm{~kg} / \mathrm{ha}^{-1}\right)$.

O nível de tecnologia empregado no cultivo de soja do Brasil, assim como as adaptações e inovações promovidas pela pesquisa, empresas e produtores rurais, desenvolveram um conjunto de práticas e conhecimentos únicos no mundo. Essas práticas permitiram a adaptação dessa espécie vegetal, originária da China (Ronzelli Jr., 1996; EMBRAPA, 2020), mais especificamente de uma região de clima subtropical úmido (Cfa) (CLIMATE-DATA.ORG, 2020), às diversas condições climáticas brasileiras, desde Cfa, no sul e sudeste, passando pelo clima de savana (Af), no centro-oeste, até o semiárido quente (BSh), do nordeste do Brasil. Esse processo de adaptação às condições edafoclimáticas nacionais ficou conhecido como Tropicalização e é um dos exemplos mais marcantes da contribuição científica brasileira à produção global de alimentos (EMBRAPA, 2018).

Em vários setores "antes da porteira” (máquinas, equipamentos, serviços e insumos utilizados nas lavouras) a cultura da soja é a majoritária em termos de relevância e inovação. De acordo com a Associação das Indústrias de Tecnologia em Nutrição Vegetal (ABISOLO, 2020), no segmento de Fertilizantes Especiais, que inclui fertilizantes foliares e biofertilizantes (conhecidos no exterior como bioestimulantes), fertilizantes orgânicos e organominerais, a cultura da soja é responsável por 49,6\% da utilização de foliares no Brasil, 7,4\% dos fertilizantes orgânicos para aplicação via solo e 30,2\% dos fertilizantes organominerais para aplicação via solo. Os dados demonstram que o sojicultor é dos produtores rurais que mais tem adotado novas tecnologias na agricultura nacional.

Devido ao grande porte do cultivo e sua crescente demanda por tecnologias inovadoras, que permitam ao produtor colher mais, em uma mesma área de produção, o número de empresas que acessam os sojicultores, por meio de distribuidores ou mesmo em atendimento direto, tem sido cada vez maior. Desse modo, a implementação de rotinas de identificação, tratamento e implantação na propriedade, de conhecimento do ambiente externo, assim como de capacidade de acesso à novas tecnologias e informações são fundamentais, para garantir a evolução do cultivo em um ambiente cada vez mais competitivo. 
Diante dos importantes números da cultura da soja no Brasil, sua relevância social, econômica e tecnológica, o maior conhecimento do sojicultor é fundamental, entendendo seu perfil, grau de escolaridade, idade, hábitos de consumo de mídia, práticas de gestão do negócio, capacidade de captura de informações e tendências do mercado, tanto em meios tradicionais quanto nos meios digitais. A correlação desses parâmetros com o resultado da atividade e geração de vantagens competitivas da propriedade pode trazer importantes guias para pesquisadores, produtores rurais e empresas privadas que atuam na cultura da soja no Brasil.

Um aspecto adicional importante é a relação entre o nível e frequência de utilização de meios digitais na vida particular dos produtores rurais, com o nível e frequência de sua utilização na vida profissional, ou seja, na atividade agrícola. Diferenças no comportamento digital dos sojicultores nos dois aspectos de sua vida podem indicar preferências, dificuldades e oportunidades para maior utilização de ferramentas digitais como fonte de informação, no apoio na gestão da atividade, na compra de insumos e venda da produção agrícola. Assim, o objetivo desta pesquisa é entender a realidade e perspectivas futuras do produtor rural em termos de busca e utilização de inovações, hábito de uso de ferramentas digitais e sua utilidade na gestão e tomada de decisão na atividade agrícola.

\section{Material e Métodos}

Para maior entendimento das características do agricultor brasileiro, em especial dos sojicultores, bem como os resultados de sua atividade, foram selecionadas duas importantes regiões produtoras de soja no Brasil, de modo a representar dois perfis de produtores típicos do cultivo. A seleção de duas regiões do Brasil visou reduzir o universo amostral e concentrar os esforços de captação de respostas para a conclusão do presente estudo. Foi selecionada a região norte e centro do estado do Paraná, que é atualmente o maior produtor de grãos do Brasil e terceiro maior produtor de soja (4,3 milhões de ha), tendo a maior produtividade média no grupo de estados brasileiros que plantam acima de 2 milhões de ha da oleaginosa (IBGE, 2020b). Também foi selecionada a região de maior crescimento do cultivo atualmente, conhecida como MATOPIBA, uma junção das siglas das quatro unidades da federação do Maranhão, Tocantins, Piauí e Bahia, que juntas englobam uma área de plantio de 3,6 milhões de ha de soja (IBGE, 2020b), em áreas com solo, clima e latitude completamente diferentes do estado do Paraná, de modo a representar dois modelos bastante diversos entre si, mas típicos da sojicultura brasileira.

A coleta de dados foi realizada através de formulário eletrônico contendo 91 perguntas. A plataforma digital utilizada foi o Office Forms, da Microsoft. O questionário foi dividido nos seguintes blocos de informações: 1. Cadastro; 2. Perfil do respondente e papel na propriedade rural; 3. Atividades agrícolas, porte e produtividade obtida; 4. Capacidades de absorção de inovação (ABS); 5. Capacidades de adaptação à inovação (ADA); 6. Capacidades para a inovação (INO); 7. Capacidades para desenvolver vantagens competitivas (VC); 8. Uso de meios digitais na vida privada (UPess); 9 . Uso de meios digitais na atividade agrícola (UFarm).

Para a divulgação do formulário de pesquisa foi utilizado o disparo de e-mails para uma lista de cerca de 500 contatos de produtores rurais. Realizou-se, também, a divulgação do link para o formulário através do aplicativo mensagens WhatsApp, por meio de grupos de engenheiros agrônomos e agricultores, das regiões alvo do estudo.

Também foram realizadas coletas de dados por meio de entrevistas telefônicas, utilizando-se o mesmo formulário de pesquisa, para que fosse possível captar respostas de produtores de diferentes perfis e que não tiveram acesso ou interesse em responder a pesquisa digitalmente.

No total, foram coletadas 55 respostas. Das respostas obtidas, 32 foram por meio de formulário eletrônico e 23 por meio de entrevistas telefônicas. Com isso, obteve-se um número suficiente e representativo das duas regiões e perfis de produtores rurais.

As hipóteses ou constructos sob estudo no trabalho foram as seguintes (Figura 1): A utilização de meios digitais na vida privada dos produtores tem relação direta e proporcional com a utilização de meios digitais na atividade agrícola (H1); A utilização de meios digitais na atividade rural tem influência positiva nas capacidades de absorção da inovação, adaptação à inovação e na capacidade de inovação da propriedade (H2); As capacidades de inovação têm influência positiva na geração de vantagens competitivas do negócio $(\mathrm{H} 3)$. 


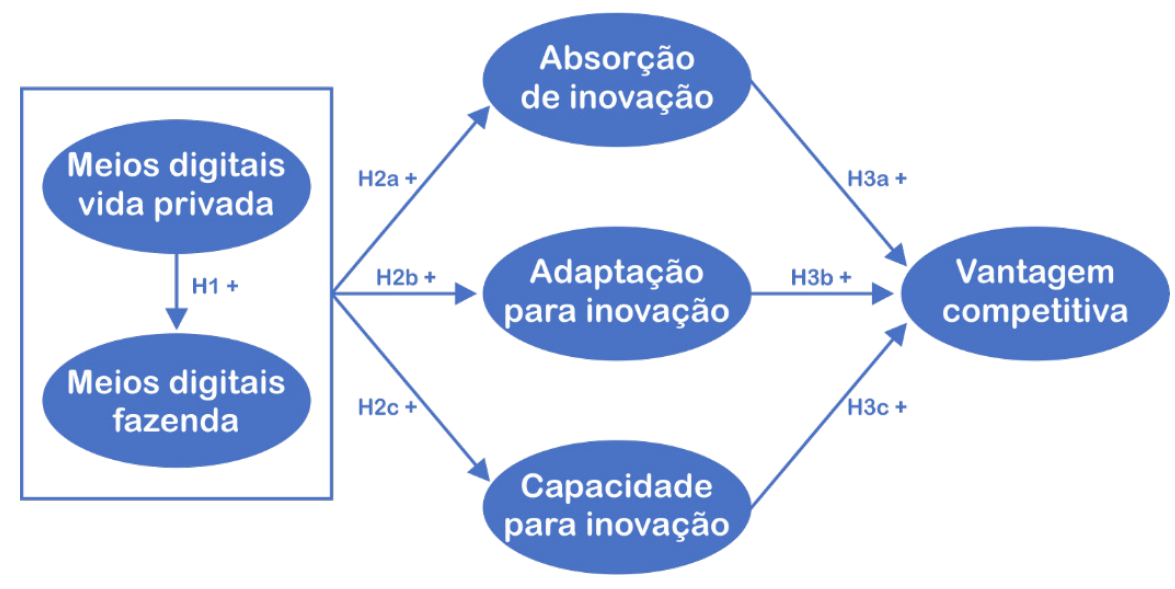

Figura 1. Modelo conceitual dos constructos ou hipóteses Fonte: Elaborado pelos autores

Visando confirmar e quantificar as hipóteses apresentadas, utilizou-se a modelagem de equações estruturais (SEM) com mínimos quadrados parciais (PLS) para mensuração da qualidade do trabalho e relacionamento entre os constructos. Utilizou-se o software SmartPLS (v3.2.6) para medições e confirmação das hipóteses (Henseler et al., 2014; Ringle et al., 2015).

Para avaliação e interpretação dos resultados coletados sobre a utilização de meios digitais pelos produtores rurais, devido ao grande número de itens desse quesito, utilizou-se, também, a análise fatorial exploratória. Segundo Aaker et al. (2004), a análise fatorial cria fatores ou grupos, combinando variáveis individuais do estudo fortemente correlacionadas entre si, facilitando o entendimento e a discussão da estrutura fundamental de um conjunto grande de variáveis.

\section{Resultados e Discussão}

As áreas de produção agrícola dos 55 agricultores entrevistados totalizaram 74.684,78 ha plantados (média de 1.357,89 ha por propriedade) e além da cultura da soja, houve destaque para o cultivo de milho (presente em 46 estabelecimentos), trigo (10 estabelecimentos), sorgo (seis estabelecimentos) e feijão (três estabelecimentos). Outras lavouras citadas, em menor número, foram abacaxi, algodão, aveia, café, cana, capim, citros, mandioca, milheto e tomate.

Especificamente para a cultura da soja, a produtividade média informada pelos produtores atingiu $60,5 \mathrm{sc} / \mathrm{ha}^{-1}\left(3.630 \mathrm{~kg} / \mathrm{ha}^{-1}\right), 7,4 \%$ acima da média nacional, mencionada anteriormente. Fica evidente que a amostra selecionada enquadrou-se em uma faixa de produtividade próxima a média nacional, o que corrobora com as regiões selecionadas e composição amostral.

Com relação ao perfil dos participantes, $90 \%$ são homens e $10 \%$ mulheres. A idade média dos produtores da amostra foi de 40,7 anos, com 54\% desses entre 35 e 49 anos de idade (Figura 2A). Com relação ao nível de instrução, 51\% possuem formação superior, $29 \%$ possuem $2^{\circ}$ grau completo, $12 \%$ possuem pós-graduação e $8 \%$ Ensino Fundamental completo (Figura 2B).

A)

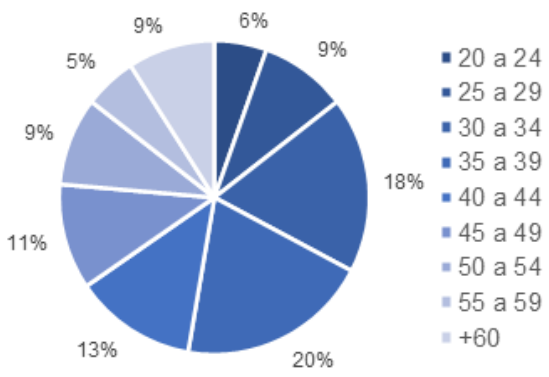

B)

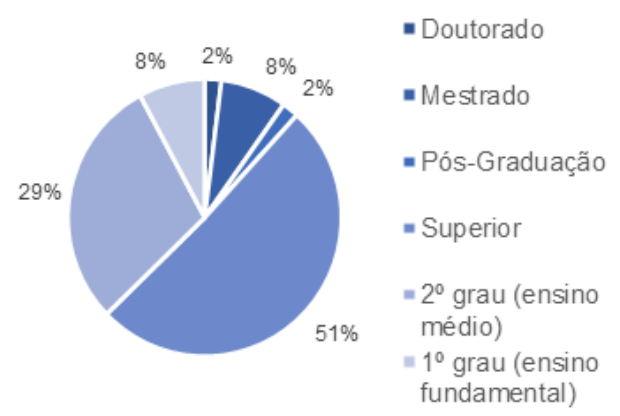


C)

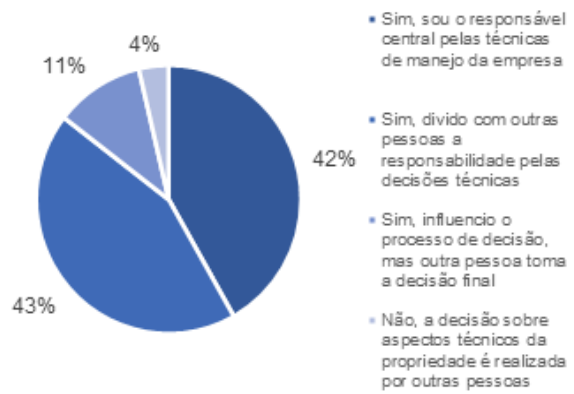

D)

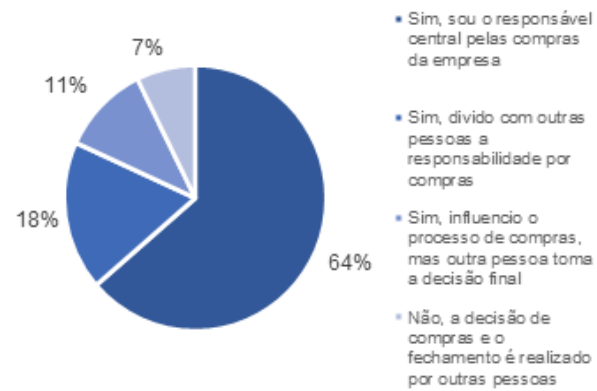

Figura 2. (A) faixa etária; (B) grau de instrução dos participantes da pesquisa; (C) envolvimento do respondente na definição das tecnologias; (D) envolvimento do respondente no processo de compras da propriedade

Fonte: Elaborado pelos autores

A amostra possui $96 \%$ dos respondentes com "responsabilidade ou influência pela definição das tecnologias empregadas na propriedade", sendo que $42 \%$ destes se identificaram como "responsáveis centrais pela tomada de decisão técnica" (Figura 2C). Já 93\% "respondem ou influenciam o processo de compras da fazenda", com $64 \%$ das pessoas identificadas como "responsáveis centrais pelas compras da propriedade" (Figura 2D). Os elevados índices apresentados nessas duas respostas confirmaram que a amostra conseguiu captar respostas de pessoas que, de fato, eram responsáveis tanto pelas práticas culturais e tecnologias adotadas quanto pela decisão de compras, ou seja, pelas tomadas de decisão mais estratégicas relacionadas ao empreendimento rural.

Com relação ao processo de digitalização ou de transformação digital, por vários ângulos tem-se observado maior pressão para a mudança, entre as quais a globalização, a maior competitividade e a entrada de competidores mais hábeis no uso dos meios digitais, disputando o mesmo espaço no mercado (MIT Center for Digital Business \& Capgemini Consulting, 2011).

Os dados coletados apresentaram informações relevantes sobre os hábitos de consumo de mídias e ferramentas digitais pelos produtores rurais das regiões estudadas, que são apresentadas nas Tabelas 1 e 2, respectivamente relativas ao uso de meios digitais na vida particular e na atividade rural (fazenda). Analisando os dados por meio de análise fatorial, foi possível agrupar os indicadores em três fatores gerais para utilização dos meios digitais nas duas esferas analisadas: Uso de Ferramentas Digitais; Digital como Fonte de Informação; e, Limitantes da Digitalização.

Por meio da interpretação das médias das respostas e dos parâmetros Top To Box (T2B) e Bottom To Box (B2B), que representaram, respectivamente, as respostas 4 e 5 (concordância) e 1 e 2 (discordância), avaliou-se que a digitalização na atividade agrícola atualmente está tão intensa ou até superior a digitalização na rotina particular dos agricultores. Observou-se que nos três fatores de agrupamento a média, o T2B e o B2B foram muito similares, com leve tendência a valores superiores para uso profissional em Uso de Ferramentas Digitais e Limitantes da Digitalização, comparado ao uso privado, com médias $5,5 \%$ e $4,7 \%$ mais elevadas, respectivamente. Em contrapartida, para o fator Digital como Fonte de Informação, a concordância média dos respondentes foi menor que 8,1\% para a atividade profissional comparada com o uso particular. Esses resultados confirmaram a grande importância dos meios digitais para gerar soluções para o negócio agrícola, e que as limitantes para maior digitalização são mais relevantes nas fazendas do que nas residências dos produtores rurais.

Tabela 1. Variáveis da pesquisa e fatores de agrupamento dos meios digitais no uso particular dos entrevistados INDICADOR DE AVALIAÇÃO $\quad$ Código Média T2B ${ }^{1}$ B2B $^{2}$

\begin{tabular}{|c|c|c|c|c|}
\hline USO DE FERRAMENTAS DIGITAIS & & 3,8 & 63 & 13 \\
\hline $\begin{array}{l}\text { Em minha rotina particular, utilizo diversas ferramentas digitais, para me } \\
\text { informar e para fazer contato com amigos e familiares. }\end{array}$ & aUPess3 & 4,3 & 87 & 5 \\
\hline $\begin{array}{l}\text { Os aplicativos para smartphone são ótimas ferramentas para me ajudar em } \\
\text { diferentes necessidades do dia a dia. }\end{array}$ & aUPess7 & 4,3 & 82 & 9 \\
\hline
\end{tabular}




\begin{tabular}{|c|c|c|c|c|}
\hline INDICADOR DE AVALIAÇÃO & Código & Média & $\mathrm{T}_{2} \mathrm{~B}^{1}$ & ${\mathrm{~B} 2 \mathrm{~B}^{2}}^{2}$ \\
\hline $\begin{array}{l}\text { Utilizo com frequência sites de compras para adquirir diversos tipos de produtos } \\
\text { e serviços. }\end{array}$ & aUPess8 & 3,7 & 65 & 18 \\
\hline $\begin{array}{l}\text { Sites de comparação de preços fazem atualmente parte de meu processo de } \\
\text { compras. }\end{array}$ & aUPess9 & 3,4 & 47 & 20 \\
\hline $\begin{array}{l}\text { Acompanho e me informo sobre temas de meu interesse particular através das } \\
\text { opiniões de Influenciadores/Formadores de opinião digitais. }\end{array}$ & aUPess10 & 2,9 & 38 & 38 \\
\hline $\begin{array}{l}\text { Quando realizo compras on-line, o que eu mais valorizo é a facilidade de acesso } \\
\text { às informações técnicas sobre o produto. }\end{array}$ & aUPess12 & 3,7 & 55 & 9 \\
\hline $\begin{array}{l}\text { O que mais valorizo nas compras on-line é a comodidade de poder comprar a } \\
\text { qualquer momento e de qualquer lugar. }\end{array}$ & aUPess13 & 3,9 & 65 & 5 \\
\hline $\begin{array}{l}\text { Utilizo os meios digitais para aquisição de pacotes de viagens, passagens aéreas } \\
\text { e reservas de hotéis. }\end{array}$ & aUPess17 & 4,0 & 71 & 5 \\
\hline $\begin{array}{l}\text { Acredito que o meio digital é adequado para compras de bens mais duráveis, } \\
\text { como veículos, ferramentas e eletrodomésticos. }\end{array}$ & aUPess18 & 3,6 & 58 & 9 \\
\hline DIGITAL COMO FONTE DE INFORMAÇÃO & & 3,9 & 70 & 11 \\
\hline $\begin{array}{l}\text { Em minha residência e locais que frequento, tenho acesso fácil e de qualidade } \\
\text { à conexão com a Internet. }\end{array}$ & cUPess1 & 4,2 & 80 & 7 \\
\hline $\begin{array}{l}\text { Sou sempre um dos primeiros a adotar novas ferramentas digitais e as utilizo } \\
\text { com alta frequência. }\end{array}$ & dUPess2 & 3,7 & 56 & 7 \\
\hline Sou usuário frequente de portais de notícias, entretenimento e curiosidades. & cUPess4 & 4,1 & 76 & 2 \\
\hline Sou usuário frequente de redes sociais (como Facebook, Instagram, entre outras). & cUPess5 & 3,7 & 67 & 22 \\
\hline $\begin{array}{l}\text { O WhatsApp é a ferramenta digital que utilizo mais vezes ao dia para assuntos } \\
\text { pessoais e de família. }\end{array}$ & cUPess6 & 4,7 & 95 & 4 \\
\hline Valorizo muito nas compras on-line o anonimato e o sigilo que elas oferecem. & dUPess14 & 3,3 & 44 & 22 \\
\hline LIMITANTES DA DIGITALIZAÇÃO & & 3,3 & 49 & 24 \\
\hline $\begin{array}{l}\text { Prefiro utilizar os meios digitais apenas para a busca de informações, } \\
\text { esclarecimento de dúvidas e comparações entre produtos, mas sempre realizo a } \\
\text { compra efetiva em pontos físicos de vendas (lojas). }\end{array}$ & bUPess11 & 3,5 & 53 & 25 \\
\hline $\begin{array}{l}\text { Valorizo as compras on-line APENAS para itens de menor valor, como alimentos } \\
\text { prontos e serviços de mobilidade/transporte urbano. }\end{array}$ & bUPess15 & 2,8 & 25 & 33 \\
\hline $\begin{array}{l}\text { Para aquisição de serviços domésticos, como de telefonia, internet e TV por } \\
\text { assinatura, os meios digitais são bastante interessantes. }\end{array}$ & bUPess16 & 3,7 & 60 & 11 \\
\hline Não acredito que os meios digitais sejam adequados para compras de imóveis. & bUPess19 & 3,5 & 57 & 22 \\
\hline $\begin{array}{l}\text { Eu só não utilizo mais meios digitais para compras, pois NÃO tenho confiança } \\
\text { de que receberei o produto conforme o anunciado. }\end{array}$ & bUPess20 & 3,0 & 42 & 38 \\
\hline $\begin{array}{l}\text { Utilizaria mais meios digitais para compras, caso houvesse um sistema de } \\
\text { atendimento sempre disponível, com uma pessoa real para tirar minhas dúvidas. }\end{array}$ & bUPess 21 & 3,6 & 65 & 16 \\
\hline $\begin{array}{l}\text { As informações das especificações dos produtos e serviços apresentados on- } \\
\text { line são geralmente INSUFICIENTES para que eu finalize a compra on-line. }\end{array}$ & bUPess22 & 3,3 & 44 & 22 \\
\hline $\begin{array}{l}\text { A dificuldade e demora na entrega de produtos para minha região, impedem } \\
\text { que eu compre mais produtos on-line. }\end{array}$ & bUPess 23 & 3,3 & 44 & 27 \\
\hline $\begin{array}{l}\text { Mesmo estando satisfeito com os sistemas digitais para compras, prefiro } \\
\text { privilegiar o comércio físico de minha cidade, mesmo que para isso pague um } \\
\text { pouco mais caro. }\end{array}$ & bUPess24 & 3,4 & 51 & 25 \\
\hline
\end{tabular}

Fonte: Elaborado pelos autores

Notas: 1 . "Top to box" representa respostas 4 e 5 , concordo na maior parte e concordo totalmente; 2 ."Bottom to box" representa respostas 1 e 2, discordo totalmente e discordo na maior parte.

Tabela 2. Variáveis da pesquisa e fatores de agrupamento dos meios digitais no uso nas atividades agrícolas dos entrevistados

\begin{tabular}{|c|c|c|c|c|}
\hline Indicador de avaliação & Código & Média & $\mathrm{T}^{\mathrm{B}} \mathrm{B}^{1}$ & ${\mathrm{~B} 2 \mathrm{~B}^{2}}^{2}$ \\
\hline USO DE FERRAMENTAS DIGITAIS & & 4,0 & 71\% & $9 \%$ \\
\hline $\begin{array}{l}\text { Utilizo diversas ferramentas digitais, para me informar e para fazer contato } \\
\text { com parceiros de negócios, fornecedores e compradores. }\end{array}$ & aUFarm3 & 4,3 & $89 \%$ & $2 \%$ \\
\hline
\end{tabular}




\begin{tabular}{|c|c|c|c|c|}
\hline Indicador de avaliação & Código & Média & $\mathrm{T}^{2} \mathrm{~B}^{1}$ & ${\mathrm{~B} 2 \mathrm{~B}^{2}}^{2}$ \\
\hline $\begin{array}{l}\text { O WhatsApp é a ferramenta digital que utilizo mais vezes ao dia no meu } \\
\text { trabalho. }\end{array}$ & aUFarm6 & 4,6 & $95 \%$ & $2 \%$ \\
\hline $\begin{array}{l}\text { Utilizo com frequência sites de compras para adquirir diversos tipos de } \\
\text { produtos e serviços para minha propriedade. }\end{array}$ & aUFarm8 & 3,1 & $35 \%$ & $29 \%$ \\
\hline $\begin{array}{l}\text { Acredito que no futuro utilizarei os meios on-line para efetivar compras de } \\
\text { insumos agrícolas. }\end{array}$ & aUFarm12 & 3,9 & $65 \%$ & $5 \%$ \\
\hline $\begin{array}{l}\text { Caso eu tenha acesso de modo fácil a todas as informações técnicas sobre o } \\
\text { insumo, é provável que eu realize compras on-line. }\end{array}$ & aUFarm13 & 3,6 & $56 \%$ & $13 \%$ \\
\hline $\begin{array}{l}\text { Para que eu realize uma compra on-line de insumos, eu preciso ter certeza de } \\
\text { que o preço será abaixo do encontrando nos pontos de vendas. }\end{array}$ & aUFarm14 & 4,2 & $80 \%$ & $5 \%$ \\
\hline $\begin{array}{l}\text { Caso haja suporte técnico disponível na minha região, no caso de algum } \\
\text { problema ocorrer com o insumo, eu sinto confiança em compras on-line. }\end{array}$ & aUFarm15 & 3,9 & $75 \%$ & $9 \%$ \\
\hline $\begin{array}{l}\text { Se a loja virtual for de um fabricante de grande porte e de alta reputação no } \\
\text { meio agrícola, eu estaria propenso a efetivar compras. }\end{array}$ & aUFarm16 & 4,0 & $73 \%$ & $5 \%$ \\
\hline DIGITAL COMO FONTE DE INFORMAÇÃO & & 3,6 & $62 \%$ & $23 \%$ \\
\hline $\begin{array}{l}\text { Em minha propriedade rural e escritório, tenho acesso fácil e de qualidade } \\
\text { à conexão com a Internet. }\end{array}$ & bUFarm1 & 4,0 & $73 \%$ & $18 \%$ \\
\hline $\begin{array}{l}\text { Sou usuário frequente de portais de notícias do Agro, informações sobre } \\
\text { manejo e análises dos mercados agrícolas, por meios digitais. }\end{array}$ & cUFarm4 & 4,1 & $84 \%$ & $4 \%$ \\
\hline $\begin{array}{l}\text { As mídias sociais (como Facebook, Instagram, entre outras) são, hoje, uma } \\
\text { fonte de informações de produtos, serviços e tecnologias para minha } \\
\text { atividade profissional. }\end{array}$ & bUFarm5 & 3,0 & $42 \%$ & $36 \%$ \\
\hline $\begin{array}{l}\text { Acompanho e me informo sobre temas técnicos e de mercados agrícolas } \\
\text { por meio das opiniões de Influenciadores/Formadores de opinião digitais. }\end{array}$ & bUFarm9 & 3,0 & $40 \%$ & $36 \%$ \\
\hline $\begin{array}{l}\text { Nunca utilizei meios digitais para efetivar uma compra de insumos agrícolas, } \\
\text { como fertilizantes, sementes e defensivos. }\end{array}$ & cUFarm11 & 4,1 & $73 \%$ & $18 \%$ \\
\hline LIMITANTES DA DIGITALIZAÇĀO & & 3,5 & $55 \%$ & $20 \%$ \\
\hline $\begin{array}{l}\text { Não utilizo tanto ferramentas digitais para minhas atividades profissionais } \\
\text { quanto utilizo na minha vida privada. }\end{array}$ & dUFarm2 & 3,4 & $51 \%$ & $35 \%$ \\
\hline Aplicativos para smartphone trazem ótimas ferramentas para meu negócio. & eUFarm7 & 4,0 & $71 \%$ & $5 \%$ \\
\hline $\begin{array}{l}\text { Utilizo os meios digitais apenas para a busca de informações, esclarecimento } \\
\text { de dúvidas e comparações entre produtos para minha produção, mas sempre } \\
\text { realizo a compra efetiva em pontos físicos (Revendas e/ou Cooperativas) }\end{array}$ & dUFarm10 & 4,1 & $82 \%$ & $7 \%$ \\
\hline $\begin{array}{l}\text { Não realizaria compras on-line de insumos agrícolas pois não tenho segurança } \\
\text { que a entrega ocorreria conforme o programado em minha região. }\end{array}$ & eUFarm17 & 2,7 & $27 \%$ & $44 \%$ \\
\hline $\begin{array}{l}\text { O fator mais importante para que eu utilize meios digitais para compra } \\
\text { de insumos é a segurança com relação a meus dados pessoais e sigilo da } \\
\text { operação. }\end{array}$ & dUFarm18 & 3,5 & $45 \%$ & $15 \%$ \\
\hline $\begin{array}{l}\text { Mesmo estando satisfeito com os sistemas digitais para compras } \\
\text { profissionais, prefiro privilegiar o comércio físico de minha cidade, mesmo } \\
\text { que para isso pague um pouco mais caro. }\end{array}$ & eUFarm19 & 3,5 & $56 \%$ & $15 \%$ \\
\hline
\end{tabular}

Fonte: Elaborado pelos autores

Notas: 1 ."Top to box" representa respostas 4 e 5, concordo na maior parte e concordo totalmente; 2 ."Bottom to box" representa respostas 1 e 2 , discordo totalmente e discordo na maior parte.

O fator Digital como Fonte de Informação evidenciou a relevância do digital como fonte de informação profissional tanto quanto de informações pessoais, com $62 \%$ dos respondentes indicando esses meios como fontes altamente relevantes em seus hábitos de mídia. Por outro lado, $23 \%$ dos respondentes indicaram baixa relevância dos meios digitais como fontes de informação agrícola relevantes. Essa relação confirmou que o produtor rural e o meio agrícola, de maneira geral, estão em fase intermediária no processo de digitalização, ainda com muitas oportunidades de crescimento e aumento da frequência de uso.

O terceiro bloco de fatores concentrou-se em Limitantes da Digitalização das propriedades como limitações logísticas para entregas, sigilo e segurança das operações on-line, e a tradição e hábito dos produtores nas compras por meio de distribuidores, como revendas e cooperativas de insumos agrícolas. Em outro estudo, McKinsey e Company (2020) também apresentaram dados que mostram que $40 \%$ dos produtores aumentariam seu envolvimento por canais digitais caso sua percepção sobre a segurança das plataformas fosse maior. 
As mudanças trazidas pela digitalização nas relações de consumo e no marketing, de maneira geral, foram amplamente discutidas por Kotler (2017). Os consumidores são muito bem-informados, estudam produtos, serviços, avaliações de outros consumidores e são muito mais ativos na busca por novas opções e tecnologias, por meio do uso intensivo da conectividade. O novo consumidor não está tão sujeito a influência ativa das grandes companhias através dos meios tradicionais de comunicação de massa. A ampla disponibilidade de informação na rede mundial resultou em um novo caminho do consumidor, mais complexo, difuso e não linear.

Com base nos dados coletados, analisando-se apenas as questões relativas às compras on-line na rotina pessoal e na atividade nas fazendas, foi possível constatar que, na média, a concordância com o uso frequente de compras on-line para a atividade agrícola foi 16,8\% menor do que na rotina pessoal, com variação negativa ainda mais expressiva no T2B, que cai de $65 \%$ para apenas $35 \%$ nas compras online frequentes para a fazenda. Há, ainda, a constatação de que $73 \%$ dos produtores declaram nunca ter realizado compras on-line de insumos agrícolas, em grande contraste com a média de $63 \%$ que declaram que os meios digitais são importantes para compras de diversos serviços e produtos para uso particular e familiar. Esses resultados comprovaram que há um grande espaço para a exploração de modalidades de vendas on-line para serviços, equipamentos e insumos agrícolas, tendo em vista que os agricultores já estão bastante familiarizados com compras on-line, em especial em sua vida privada.

McKinsey e Company (2020) também apresentaram informações sobre o uso de meios digitais para compras nas propriedades agrícolas brasileiras. 0 estudo mostrou que $36 \%$ dos agricultores realizaram compras on-line para a atividade, porém sem especificar se as referidas compras foram de insumos agrícolas ou outros itens, de ligação mais secundária com o manejo dos cultivos. Este estudo, por sua vez, também mostrou que $33 \%$ dos entrevistados estão dispostos a comprar sementes, fertilizantes e defensivos on-line - nas próximas duas safras -, com percentuais mais elevados para produtores de grãos do MATOPIBA e menores para produtores de grãos do sul do país. Neste estudo, concluiu-se que $27 \%$ dos produtores já realizaram alguma compra de insumos agrícolas por vias digitais, mostrando boa correlação com os dados apresentados no trabalho aqui referenciado.

No entanto, alguns fatores foram apresentados como críticos para maior propensão a compras online, em especial de insumos agrícolas, como: i) a garantia de que os preços sejam mais baixos do que os disponíveis em canais usuais de compras; ii) que as lojas virtuais sejam de empresas de grande porte e boa reputação; iii) que seja garantido suporte técnico presencial, no caso de algum problema com o produto; iv) que as informações sejam claras, completas e transparentes e; v) que haja uma boa logística de entrega dos insumos na região.

Também com relação a compras on-line de insumos, na questão de número 91 (qualitativa) houve menções frequentes sobre o grande potencial das compras on-line de insumos num futuro próximo e que, para isso, vários fatores precisam melhorar e ser oferecidos ao produtor rural, como condições de pagamento a prazos usuais do mercado agrícola, logística ágil, assistência técnica e preços vantajosos.

As limitantes para compras on-line de insumos apresentadas pelos respondentes parecem indicar que o setor agrícola terá que encontrar um meio próprio e único de digitalização dos processos de compras. Esse novo modelo provavelmente passará por forte interligação e combinação de mecanismos on-line e off-line no caminho do consumidor, desde a assimilação, atração, arguição, ação, até a fase da apologia. No caminho, meios digitais e físicos foram combinados e complementados, resultando no processo onicanal, como citado por Kotler (2017).

As observações quantitativas, relacionadas a fatores limitantes para maior uso de meios digitais nas fazendas, foram também corroboradas nas respostas qualitativas coletadas na questão de número 91 do instrumento de pesquisa. Houve uma menção espontânea significativa de dificuldade de acesso à conexão de dados de boa qualidade nas áreas produtivas, o que impõe limites importantes para implantação de sistemas, bem como para controle e monitoramento remoto de atividades, por exemplo.

Para comprovar as outras duas hipóteses do estudo, ou seja, a influência positiva dos meios digitais utilizados na propriedade na capacidade de absorção da inovação, na adaptação à inovação e na capacidade de inovação, assim como a influência positiva da capacidade de inovação na geração de vantagens competitivas da propriedade, realizou-se diferentes análises no modelo de medição, de acordo com a natureza de cada constructo, reflexivo ou formativo. O modelo reflexivo foi aplicado, retornando com relações confiáveis para as variáveis ABS, INO e VC. Para esses constructos, todas as variáveis mostraram boa consistência interna com alfa na escala de Cronbach's entre 0,725 e 0,899 e fiabilidade composta (FC) entre 0,840 e 0,899. Também a variância média extraída (AVE) foi superior à 0,5, conforme apresentado na Tabela 3, confirmando a validade do modelo. 
Tabela 3. Modelo de medição reflexiva

\begin{tabular}{lcc} 
Constructos & Fiabilidade composta (FC) & Variância Média Extraída (AVE) \\
Capacidade para absorção (ABS) & 0,899 & 0,641 \\
Capacidade para inovação (INO) & 0,925 & 0,712 \\
Vantagens competitivas (VC) & 0,840 & 0,638 \\
\hline
\end{tabular}

Fonte: Elaborado pelos autores

As avaliações realizadas confirmaram que os constructos representaram uma dimensão independente e que foram capazes de explicar mais da metade da variância de seus indicadores. 0 modelo apresentou boa confiabilidade, conforme apresentado na Tabela 4, que demonstrou análises de carregamento satisfatórias. Também é apresentado na Tabela 5 o teste de validade discriminante Fornell-Larcker (1981). Os dois métodos indicaram que o estudo tem boa validade discriminante para explicar os constructos.

Tabelas 4. Carregamentos PLS e cruzados

\begin{tabular}{|c|c|c|c|c|}
\hline Constructos & ABS & INO & VC & UFarm \\
\hline \multicolumn{5}{|c|}{ Capacidade para absorção (ABS) } \\
\hline ABS1 & 0,78 & & & \\
\hline ABS2 & 0,83 & & & \\
\hline ABS3 & 0,85 & & & \\
\hline ABS4 & 0,73 & & & \\
\hline ABS5 & 0,80 & & & \\
\hline \multicolumn{5}{|c|}{ Capacidade para inovação (INO) } \\
\hline INO1 & & 0,82 & & \\
\hline INO2 & & 0,81 & & \\
\hline INO3 & & 0,84 & & \\
\hline Constructos & ABS & INO & VC & Ufarm \\
\hline \multicolumn{5}{|c|}{ Vantagens competitivas (VC) } \\
\hline VC3 & & & 0,77 & \\
\hline VC4 & & & 0,90 & \\
\hline VC9 & & & 0,72 & \\
\hline \multicolumn{5}{|c|}{ Meios digitais na fazenda (Ufarm) } \\
\hline aUFarm12 & & & & 0,23 \\
\hline aUFarm13 & & & & 0,19 \\
\hline aUFarm15 & & & & 0,23 \\
\hline aUFarm16 & & & & 0,15 \\
\hline aUFarm3 & & & & 0,47 \\
\hline aUFarm6 & & & & 0,20 \\
\hline bUFarm9 & & & & 0,02 \\
\hline cUFarm4 & & & & 0,60 \\
\hline dUFarm10 & & & & 0,63 \\
\hline dUFarm18 & & & & 0,31 \\
\hline eUFarm7 & & & & 0,23 \\
\hline
\end{tabular}

Fonte: Elaborado pelos autores 
Tabela 5. Modelo de validade discriminante (critério de Fornell-Larcker, 1981) variância média extraída e correlações de variáveis latentes

\begin{tabular}{lcrrrr} 
& ABS & INO & Ufarm & VC \\
\hline Capacidade para absorção (ABS) & 0,801 & & & & \\
Capacidade para inovação (INO) & 0,718 & 0,844 & & \\
Meios digitais na fazenda (Ufarm) & 0,518 & 0,597 & Formativo & \\
Vantagens competitivas (VC) & 0,505 & 0,638 & 0,495 & 0,799 \\
\hline
\end{tabular}

Fonte: Elaborado pelos autores

O fator de utilização de meios digitais nas fazendas pode ser classificado como um fator formativo (Tabela 5). Fatores formativos constroem o constructo e, assim, a somatória das variáveis estudadas quanto à utilização de meios digitais nas fazendas formam o construto utilização de meios digitais (Ufarm). Para a avaliação do modelo formativo, uma análise de colinearidade foi realizada. As avaliações e o Variance Inflation Factor (VIF) mostraram-se abaixo da linha de corte de 3,3 (Sarstedt et al., 2014) são apresentados na Tabela 6.

Tabela 6. Avaliação de colinearidade

\begin{tabular}{|c|c|}
\hline Constructos & Variance Inflation Factor (VIF) \\
\hline ABS1 & 2,395 \\
\hline ABS2 & 2,763 \\
\hline ABS3 & 2,429 \\
\hline ABS4 & 1,967 \\
\hline ABS5 & 1,794 \\
\hline INO1 & 2,179 \\
\hline INO2 & 2,497 \\
\hline INO3 & 2,778 \\
\hline INO4 & 3,155 \\
\hline INO5 & 3,133 \\
\hline VC3 & 1,420 \\
\hline VC4 & 1,543 \\
\hline VC9 & 1,368 \\
\hline aUFarm12 & 3,828 \\
\hline aUFarm13 & 3,178 \\
\hline aUFarm15 & 3,525 \\
\hline aUFarm16 & 2,683 \\
\hline aUFarm3 & 1,933 \\
\hline aUFarm6 & 1,721 \\
\hline bUFarm9 & 1,887 \\
\hline cUFarm4 & 1,595 \\
\hline dUFarm10 & 1,410 \\
\hline dUFarm18 & 1,649 \\
\hline eUFarm7 & 1,502 \\
\hline
\end{tabular}

Fonte: Elaborado pelos autores

Após validar que o modelo de medição possuia boas propriedades psicométricas, criou-se o modelo de equações estruturais. 0 modelo, então, indicou 41\% de variação nas vantagens competitivas (Tabela 6). A significância das relações internas do modelo foi calculada por meio da aplicação de um procedimento "bootstrapping", gerando 499 amostras aleatórias (Hair Jr. et al., 2014).

O modelo e análise apresentados na Tabela 7 também mostram que a utilização de meios digitais na fazenda explica a absorção da inovação $\left(=0,518^{* * *}\right)$ e a capacidade de inovação $\left(=0,597^{* * *}\right)$. Mostram, ainda, que a capacidade de inovação explica a geração de vantagens competitivas $\left(=0,569^{* * *}\right)$. 


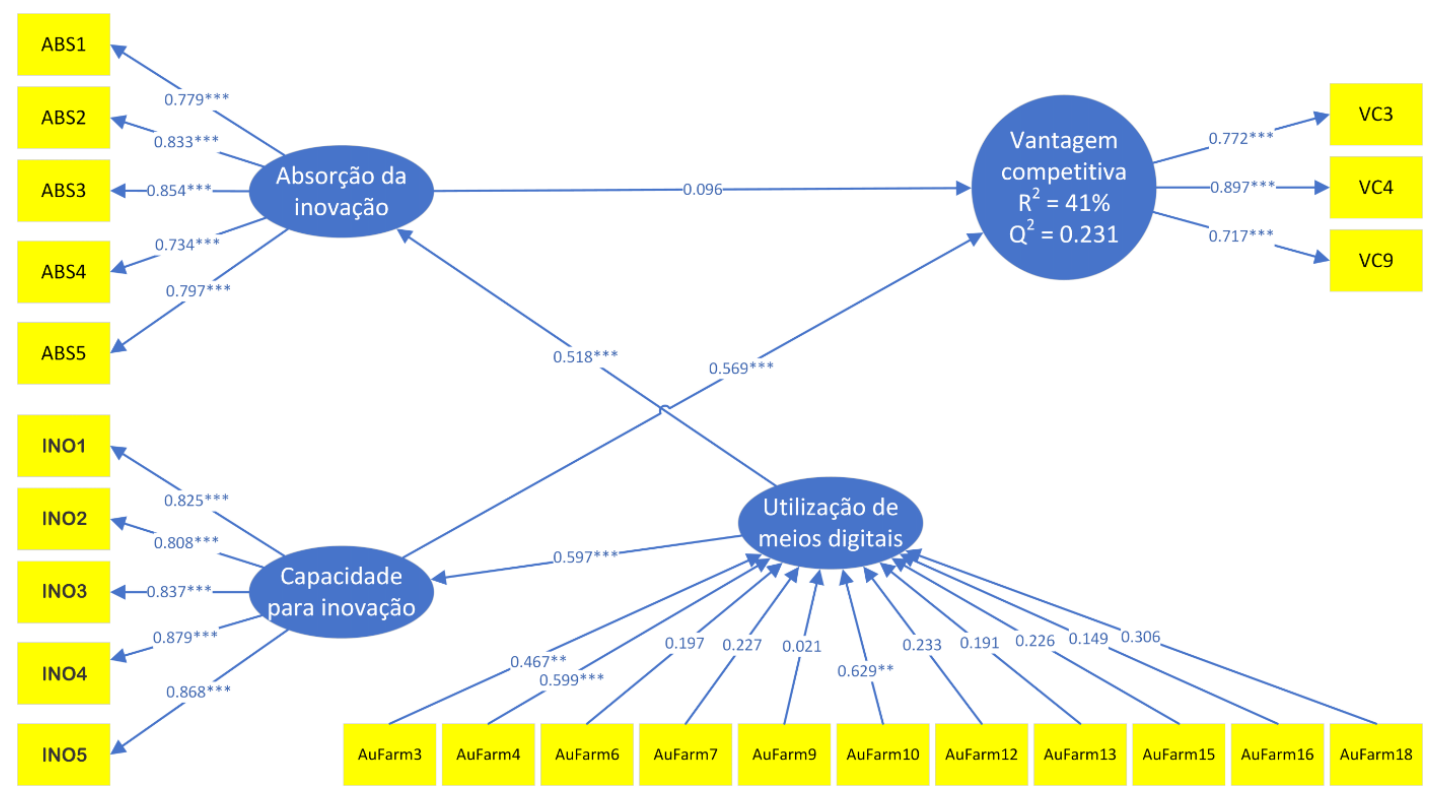

Figura 6. Modelo estrutural da pesquisa para fatores de influência na geração de vantagens competitivas das propriedades rurais

Tabela 7. Análise das hipóteses

$\mathrm{H} 1(+)$ : Uso de meios digitais na vida privada (Upess) tem influência positiva no uso de meios digitais $\mathrm{NI}$ na fazenda (Ufarm).

$\mathrm{H} 2 \mathrm{a}(+)$ : Uso de meios digitais na fazenda (Ufarm) tem influência positiva na capacidade de absorção da inovação (ABS).

$V\left(\beta=0,518^{* * *}\right)$

$\mathrm{H} 2 \mathrm{~b}(+)$ : Uso de meios digitais na fazenda (Ufarm) tem influência positiva na capacidade de adaptação à inovação (ADP). $\mathrm{H} 2 \mathrm{c}(+)$ : Uso de meios digitais na fazenda (Ufarm) tem influência positiva na capacidade para a $\mathrm{V}\left(\beta=0,597^{* * *}\right)$
inovaça (INO).

H3a(+): Capacidade de absorção da inovação (ABS) tem influência positiva na vantagem competitiva (VC). $\mathrm{NI}(\beta=0,096)$

$\mathrm{H} 3 \mathrm{~b}(+)$ : Capacidade de adaptação à inovação (ADP) tem influência positiva na vantagem competitiva (VC). NI

$\mathrm{H} 3 \mathrm{c}(+)$ : Capacidade para a inovação (INO) tem influência positiva na vantagem competitiva (VC). $\quad \mathrm{V}\left(\beta=0,569^{* * *}\right)$

Fonte: Elaborado pelos autores

Notas: $p<0.10^{*}, p<0.05^{* *}, p<0.01{ }^{* * *}$ V=Validado, NV=Não Validado, NI=Não Influente

Por meio das análises, observou-se que o Ufarm teve influência positiva na capacidade de INO. 0 modelo demonstrou que há boa correlação de $0,597^{* * *}$, muito significativa, o que indica que a cada $10 \%$ de incremento no uso de meios digitais nas propriedades rurais há incremento de $5,97 \%$ na capacidade de inovação do estabelecimento. Essa conclusão traz uma contribuição relevante e inédita sobre a relação positiva entre o uso de meios digitais e a inovação, sendo importante o aprofundamento e detalhamento maior dessa relação em futuros estudos.

A disponibilidade de internet de qualidade e o uso intensivo de diversas ferramentas digitais expõe os produtores rurais a técnicas, equipamentos e insumos inovadores, em tempo real, de qualquer parte do mundo. Conforme apresentado pela Associação Brasileira de Marketing Rural e Agronegócio (ABMRA, 2017), em pesquisa realizada com 2.090 agricultores, de quinze estados brasileiros, houve avanço na utilização de internet pelos produtores no período entre 2013 e 2017, chegando a 42\% de produtores com acesso diário a rede mundial. Mais recentemente, o estudo conduzido por McKinsey e Company (2020) e realizado com 750 produtores, de onze estados diferentes, evidenciou que $71 \%$ dos produtores utilizam canais digitais diariamente para questões ligadas à agricultura. 0 crescimento expressivo observado no uso de meios digitais pelos agricultores, de 2013 para 2017, e depois para 2020, tem trazido mais acesso a busca, estudo, implementação e adaptação de novas ferramentas para o negócio rural, completando os critérios de formação da INO proposta neste estudo. 
O Ufarm também se mostrou como fator de influência positiva na capacidade de ABS. Nesse caso, cada $10 \%$ de aumento no uso de meios digitais resultou em incremento de $5,18 \%$ na capacidade de absorção da inovação nas fazendas. Entende-se, assim, que os meios digitais, destacadamente aplicativos de trocas de mensagens, como o WhatsApp, aumentaram e facilitaram o fluxo interno de informações coletadas no ambiente externo entre os colaboradores, criando canais dinâmicos de compartilhamento, discussão e decisão sobre essas as inovações, algo que anteriormente não ocorria de modo rotineiro na maior parte dos empreendimentos agrícolas. $O$ uso intensivo do aplicativo WhatsApp foi comprovado pela nota média 4,6, a mais elevada obtida por este estudo, apresentada no item de pesquisa Ufarm6, indicando o aumento da comunicação interna promovido pela ferramenta digital nas propriedades.

O estudo não confirmou a influência positiva da maior capacidade de absorção de ABS na geração de VC, mas sim das INO em VC. Entende-se que isso se deve, em parte, à centralização das decisões no proprietário ou em um grupo muito restrito de pessoas, uma característica marcante das propriedades rurais brasileiras. Haberli e Spers (2014), em trabalho que propôs a segmentação de produtores rurais baseada em seu estilo de vida, também concluíram que existe certa onipotência no dia a dia do agricultor, pois em sua propriedade ele tudo decide, gerando individualismo na gestão do negócio. Em suma, não há, de maneira geral, rotinas claras de captação, discussão e implementação de conhecimentos na propriedade (fatores do grupo de absorção de inovação), mas há iniciativa dos patriarcas que gera testagem e aprendizagem nas propriedades, mesmo sem a rotinização do processo.

O investimento em inovação e busca por melhores práticas dentro das fazendas pode ser explicado pelo aumento da complexidade e competitividade do meio agrícola, processo que é catalisado pela maior disponibilidade de informações e tecnologias trazidas pelos meios digitais, apesar da falta de processos internos específicos para tal.

A INO foi fator determinante no aumento das VC do negócio agrícola, mostrando que a cada 10\% de aumento na capacidade para inovação houve impacto de 5,69\% nas vantagens competitivas do negócio. Dentro dos fatores relacionados à capacidade para inovação destaca-se o INO4 ("geramos inovações que substituem práticas tradicionais de nosso sistema de produção") com 0,879*** de influência, indicando que o produtor rural tem alto nível de abertura para mudanças geradas na propriedade em testes internos, implantando rapidamente inovações que têm impacto no resultado das lavouras. O perfil empreendedor e inovador do produtor rural foi também apresentado por Haberli e Spers (2014), que, com base na descrição dos perfis apresentados em seu trabalho, observaram a prevalência de perfis com disposição à inovação e experimentação e importante percentual de perfis empreendedores dentro do grupo de 257 produtores rurais pesquisados.

Analisando os dados, preconizou-se que o perfil do produtor rural que possui caraterísticas mais empreendedoras, de experimentação e de pragmatismo, pode ser um fator importante para a construção de vantagens competitivas e, consequentemente, para performances acima da média, relações essas que podem ser estudadas com maior detalhe em trabalhos futuros.

Os resultados apresentados foram relevantes e inovadores, considerando-se que poucos estudos tiveram como objetivo estudar a correção entre a maior utilização de meios digitais nas fazendas e a geração de inovação, assim como o impacto das inovações na geração de vantagens competitivas das propriedades, de modo quantitativo e mensurável. Os resultados positivos entre os constructos indicaram que diversos agentes do meio agrícola, como órgãos de pesquisa e extensão rural, órgãos reguladores - como o Ministério da Agricultura, Pecuária e Abastecimento (MAPA) - órgãos consultores e empresas de tecnologia para o meio agrícola devem, de modo decisivo, investir em meios digitais para que a informação gerada seja acessada mais facilmente e rapidamente pelos produtores rurais.

Ficou bastante evidente, também, que há restrições importantes a maior digitalização da atividade agrícola nas regiões do estudo e, de modo geral, no Brasil. Entre elas, destaca-se, com base nas respostas à pesquisa: i) problemas de cobertura de sinal de dados nas regiões agrícolas estudadas; ii) falta de confiança para aquisição de insumos nas vias digitais em função da inexperiência e falta de mecanismos de suporte técnico disponíveis e amplamente divulgados e; iii) preocupações com a segurança de dados e das transações realizadas por meios digitais, pois não há clareza de como as informações serão utilizadas pelos desenvolvedores dos sistemas digitais. 
As dificuldades relatadas pelos entrevistados indicaram que o avanço mais pronunciado dos meios digitais no campo depende fortemente de investimentos em infraestrutura de rede e em sistemas híbridos, que combinem ferramentas digitais objetivas, rápidas e com termos de uso claros, com assistência técnica presencial disponível para esclarecimento de dúvidas e atendimento a questões de qualidade dos insumos, além de uma logística funcional.

O estudo apresentou algumas limitações, que são fatores de melhoria para estudos similares no futuro. É necessário um número amostral superior para a confirmação de outros constructos, como a capacidade de adaptação para inovação e outros itens das vantagens competitivas. Novos estudos devem, também, aprofundar-se nas relações entre o perfil dos tomadores de decisão com os indicadores do estudo. Como exemplos importantes temos: a região da fazenda, o tamanho da propriedade, o grau de profissionalização do empreendimento, a idade, a escolaridade e o perfil comportamental dos produtores.

\section{Conclusão}

O acelerado crescimento e a relevância do uso de meios digitais pelos sojicultores brasileiros, tanto na vida pessoal quanto profissional, assim como sua disposição para a maior digitalização da atividade e, consequentemente, uma transformação digital mais pronunciada do agronegócio brasileiro, são comprovadas pelo estudo. Os fatores limitantes mais importantes para a maior digitalização das fazendas estão ligados a infraestrutura de redes, suporte técnico disponível, segurança das operações on-line e falta de disponibilidade de plataformas acessíveis e claras. A relação positiva entre a utilização dos meios digitais nas propriedades rurais estudadas, acelerando-se o acesso e o desenvolvimento de inovações, assim como a geração de vantagens competitivas do negócio, é quantificada e confirmada por meio de análises estatísticas fatoriais e de equações estruturais. Os resultados fornecem informações inéditas e importantes para embasar projetos de digitalização do agronegócio, assim como elementos de fundamentação para futuros estudos sobre o tema no Brasil.

Contribuição dos autores: Concepção: Ribeiro, A.N.; Haberli Jr., C. Coleta de dados: Ribeiro, A.N. Análise dos dados: Ribeiro, A.N. Definição da metodologia: Ribeiro, A.N.; Haberli Jr., C. Redação e edição: Ribeiro, A.N.; Haberli Jr., C.

Agradecimentos: Agradeço a Microquimica Tradecorp, que apoiou a realização deste estudo.

Como citar: Ribeiro, A.N.; Haberli Jr., C. 2021. Uso de Meios digitais, capacidades de inovação e resultados do sojicultor no PR e MATOPIBA. Quaestum 2: e26750584.

\section{Referências}

Aaker, D.A.; Kumar, V.; Day, G.S. 2004. Pesquisa de Marketing. 2ed. Atlas, São Paulo, SP, Brasil.

Associação Brasileira de Marketing Rural e Agronegócio (ABMRA). 2017. $7^{\text {a }}$ Pesquisa Hábitos do Produtor Rural. Disponível em: <http://www.webrural.com.br/bfd_download/7a-pesquisa-habitos-do-produtor-rural-2017fonte-associacao-brasileira-de-marketing-rural/>. Acesso em: out. 17, 2020.

Associação Brasileira das Indústrias de Tecnologia em Nutrição Vegetal (ABISOLO). 2020. $6^{\circ}$ Anuário Brasileiro das Indústrias de Tecnologia em Nutrição Vegetal 2020. Disponível em: <https://www.abisolo.com.br/anuario/>. Acesso em: ago. 03, 2020.

Companhia Nacional de Abastecimento (CONAB). 2020. Boletim da Safra de Grãos. Boletim de setembro de 2020. Disponível em: <https://www.conab.gov.br/info-agro/safras/graos/boletim-da-safra-de-graos>. Acesso em: out. 06, 2020.

Confederação da Agricultura e Pecuária do Brasil (CNA). 2020. Panorama do Agro. Disponível em: <https://www. cnabrasil.org.br/cna/panorama-do-agro\#_ftn1>. Acesso em: out. 08, 2020.

Empresa Brasileira de Pesquisa Agropecuária (EMBRAPA). 2018. Artigo: Ciência, o adubo da agricultura brasileira. Disponível em: <https://www.embrapa.br/busca-de-noticias/-/noticia/37402973/artigo-ciencia-o-adubo-daagricultura-brasileira>. Acesso em: out. 17, 2020.

Empresa Brasileira de Pesquisa Agropecuária (EMBRAPA). 2020. Embrapa Soja, História da soja. Disponível em: <https://www.embrapa.br/soja/cultivos/soja1/historia>. Acesso em: out. 08, 2020.

Haberli Jr, C.; Spers, E.E. 2014. Segmentação de produtores rurais baseada em estilo de vida: uma aplicação no mercado de fertilizantes. Desafio Online, 1(2): 599.

Hair Jr., J.F.; Sarstedt, M.; Hopkins, L.; Kuppelwieser, V.G. 2014. Partial least squares structural equation modeling (PLS-SEM): An emerging tool in business research. Europian Business Review, 26(2). https://doi.org/10.1108/ EBR-10-2013-0128.

Henseler, J; Ringle, C.M.; Sarstedt, M. 2014. A new criterion for assessing discriminant validity in variance-based structural equation modeling. J. Acad. Marketing Sci., 43(1): 115-135.

Instituto Brasileiro de Geografia e Estatística (IBGE). 2017. Censo Agropecuário 2017. Disponível em: <https://www. ibge.gov.br/estatisticas/economicas/agricultura-e-pecuaria/21814-2017-censo-agropecuario.html $?=\& \mathrm{t}=0$ que-e>. Acesso em: out. 06, 2020.

Instituto Brasileiro de Geografia e Estatística (IBGE). 2020a. Levantamento Sistemático da Produção Agropecuária 
(LSPA). Relatório de Setembro de 2020. Disponível em: <https:/www.ibge.gov.br/estatisticas/economicas/ agricultura-e-pecuaria/9201-levantamento-sistematico-da-producao-agricola.html $?=\& \mathrm{t}=0$-que-e>. Acesso em: out. 17, 2020

Instituto Brasileiro de Geografia e Estatística (IBGE). 2020b. Sistema IBGE de Recuperação Automática (SIDRA). Relatório de Setembro de 2020. Disponível em: <https://sidra.ibge.gov.br/tabela/6588>. Acesso em: out. 17, 2020.

Kotler, P. 2017. Marketing 4.0 - Do Tradicional ao Digital. GMT Editores, Rio de Janeiro, RJ, Brasil.

Massachusetts Institute of Technology (MIT) Center for Digital Business \& Capgemini Consulting. 2011. DIGITAL TRANSFORMATION: A Roadmap for Billion-Dollar Organizations. Disponível em: <https://www.capgemini. com/wp-content/uploads/2017/07/Digital_Transformation_A_Road-Map_for_Billion-Dollar_Organizations. pdf>. Acesso em: out. 02, 2020.

McKinsey \& Company. 2020. A mente do Agricultor Brasileiro na Era Digital. Disponível em: <http://www. aeaprcuritiba.com.br/admin/arquivos/A\%20mente\%20do\%20Agricultor\%20Brasileiro\%20na\%20Era\%20 Digital\%20[AGCO].pdf >. Acesso em: out. 09, 2020.

República da China Clima (CLIMATE-DATA.ORG). 2020. Disponível em: <https://pt.climate-data.org/asia/ republica-da-china-190/> Acesso em: out. 17, 2020.

Ringle, C.M.; Wende, S.; Becker, J.M. 2015. “SmartPLS 3.” Boenningstedt: SmartPLS GmbH. Disponível em: <http:// www.smartpls.com>. Acesso em: out. 09, 2020.

Ronzelli Júnior, P. 1996. Melhoramento Genético de Plantas. Graffice Editora Gráfica, Curitiba, PR, Brasil.

Sarstedt, M.; Ringle, C.M.; Smith, D.; Reams, R., Hair, J.F. 2014. Partial least squares structural equation modeling (PLS-SEM): a useful tool for family business researchers. J. Family Bus. Strategy, 5(1): 105-115.

United States Department of Agriculture (USDA). 2020a. World Agricultural Production. Circular Series WAP 1020 October 2020. Disponível em: <https://apps.fas.usda.gov/psdonline/circulars/production.pdf>. Acesso em: out. 07,2020

United States Department of Agriculture (USDA). 2020b. Export Sales Query System. Disponível em: <https:// apps.fas.usda.gov/esrquery/>. Acesso em: out. 07, 2020. 